\title{
Respostas cardiovasculares agudas de três protocolos de exercício resistido em idosas
}

\author{
Acute cardiovascular response of older women to three resistance \\ exercise protocols
}

\author{
Rodrigo Pereira da Silva ${ }^{1}$ \\ Jefferson da Silva Novaes 2 \\ Ricardo Jacó de Oliveira ${ }^{3}$ \\ Flávio de Jesus Camilo ${ }^{4}$ \\ Martim Francisco Bottaro Marques ${ }^{3}$
}

1. Universidade Federal de Ouro Preto. Ouro Preto. MG. Brasil.

2. Universidade Federal do Rio de Janeiro. Rio de Janeiro. RJ. Brasil.

3. Universidade de Brasília. Brasília. DF. Brasil.

4. Faculdades Unidas do Norte de Minas. Montes Claros. MG. Brasil.

Recebido em 19/02/09 Revisado em 25/03/09 Aprovado em 18/05/09
Resumo - As respostas cardiovasculares agudas foram comparadas entre três diferentes protocolos de treinamento resistido de alta velocidade de contração muscular em mulheres idosas destreinadas. Doze voluntárias, aparentemente saudáveis (62.6 $\pm 2,9$ anos), realizaram três diferentes protocolos no supino horizontal (SH) e no leg press $45^{\circ}$ (LP). Todos os protocolos envolveram três séries de 10 repetições realizados com uma carga de 10RM e 2 minutos de recuperação entre as séries. O protocolo contínuo consistiu em 10 repetições, com nenhuma pausa entre as repetições. Os protocolos descontínuos foram realizados com uma pausa de cinco (PD5) ou 15 (PD15) segundos, entre a quinta e sexta repetições. Frequência cardíaca (FC), pressão arterial sistólica (PAS) e duplo produto (DP) foram avaliados no repouso e ao final de todas as séries nos exercícios. ANOVA fatorial foi usada para comparar as respostas cardiovasculares entre os diferentes protocolos. Comparado com o repouso, FC, PAS e DP foram respectivamente 22.3\%, 23.2\% e 51.2\% ( $\mathrm{p}<0.05)$ maiores no exercício $\mathrm{SH}$ e $41.7 \%$, 43.0\% e 102.9\% ( $<$ < 0.05) maiores no exercício LP, após a terceira série, em todos os protocolos. FC e DP foram 5.6\% e 8.2\% ( $\mathrm{p}<0.05)$, respectivamente, menores no DP5 e DP15 comparado com o PC, no exercício SH. A FC, PAS e DP foram 5.2\%, 8.0\% e $14,8 \%$,respectivamente, menores no DP5 comparado com o PC, no exercício LP. Portanto, parece que o exercício resistido de alta velocidade descontínuo tem uma menor demanda cardiovascular do que exercício resistido contínuo em mulheres idosas.

Palavras-chave: Freqüência cardíaca; Pressão arterial; Duplo produto; Exercício resistido.

Abstract - Acute cardiovascular responses to different high-velocity resistance exercise protocols were compared in untrained older women. Twelve apparently healthy volunteers (62.6 \pm 2.9 years) performed three different protocols on the bench press (BP) and leg press (LP). All protocols consisted of three sets of 10 repetitions performed with a 10RM load and 2 min of rest between sets. The continuous protocol (CP) consisted of 10 repetitions with no pause between repetitions. The discontinuous protocols were performed with a pause of five (DP5) or 15 (DP15) seconds between the fifth and sixth repetition. Heart rate (HR), systolic blood pressure (SBP), and rate pressure product (RPP) were assessed at baseline and at the end of all exercise sets. Factorial ANOVA was used to compare the cardiovascular response among different protocols. Compared to baseline, HR, SBP and RPP were, respectively, $22.3 \%, 23.2 \%$ and $51.2 \%(p<$ $0.05)$ higher for BP exercise, and $41.7 \%, 43.0 \%$ and $102.9 \%(p<0.05)$ higher for LP exercise after the third set in all protocols. For BP exercise, HR and RPP were 5.6\% and $8.2 \%(p<$ $0.05)$ lower in DP5 and DP15, respectively, compared to CP. For LP exercise, HR, SBP and RPP were, respectively, $5.2 \%, 8.0 \%$ and $14.8 \%$ lower in DP5 compared to CP. In conclusion, discontinuous high-velocity resistance exercise seems to have a lower cardiovascular demand than continuous resistance exercise in older women.

Key words: Heart rate; Blood pressure; Double product; Resistance exercise. 


\section{INTRODUÇÃO}

O envelhecimento provoca reduções significativas na quantidade de massa corporal e força muscular, provocando incapacidade funcional ${ }^{1}$. O declínio da força muscular é devido, em maior parte, a redução da fibra muscular tipo II (contração rápida) que a do tipo I (contração lenta), o que acarreta na incapacidade de gerar potência muscular ${ }^{2,3}$.

O exercício resistido (ER), especialmente, o treinamento de potência, é uma importante estratégia para se promover adaptações fisiológicas contrárias ao envelhecimento, como aumento da massa corporal, força muscular e potência ${ }^{4}$. Entretanto, quando o ER é prescrito para a população idosa é de suma importância o monitoramento das respostas cardiovasculares agudas impostas por esse tipo de treinamento, visando à segurança do praticante. Isto se deve em virtude dos significativos aumentos na frequência cardíaca (FC) e pressão arterial (PA).

Nesse sentido, as adaptações agudas no exercício resistido (ER) vêm sendo foco de investigação para um melhor entendimento da sobrecarga cardiovascular imposta pelo exercício ${ }^{5,7}$. McCartney $^{8}$ aponta a necessidade da monitorização das variáveis fisiológicas FC e PA para maior segurança na prescrição do ER. Contudo, essas duas variáveis isoladas podem não ser suficientes para uma maior segurança. $\mathrm{O}$ duplo produto (DP) parece ser um bom indicador da sobrecarga cardíaca imposta pelo treinamento resistido?.

Tal assunto vem culminando no aparecimento de estudos com desenhos metodológicos que visam esclarecer o comportamento agudo das variáveis $\mathrm{FC}$, PA e DP no ER em adultos jovens ${ }^{5,10,11}$ e idosos ${ }^{12}$. Nesses estudos, destacam-se os de protocolos contínuos (PC), descontínuos (PD) e fracionados (PF). Entende-se por PC uma série realizada no ER sem interrupções durante a mesma, e PD e PF quando se adota uma pausa durante a execução da série. Veloso et al. ${ }^{13}$, sem controlar a velocidade de contração muscular, verificaram menores valores de FC, PAS e DP quando o protocolo de ER fracionado foi aplicado em mulheres idosas. Já Coelho et al. ${ }^{10}$, aplicando alta velocidade de contração muscular, três segundos para cada repetição, reportaram um aumento agudo significativo na FC, no protocolo contínuo de 12 repetições máximas (12-RM), quando comparado com o protocolo descontínuo ( 2 x 6 repetições), com 15 segundos de pausa entre a sexta e a sétima repetição, em uma amostra de adultos jovens.

Bottaro et al. ${ }^{4}$, utilizando um protocolo contínuo, reportaram que 10 semanas de treinamento resistido de potência melhora a capacidade funcional e a potência muscular em idosos. Porém, existe uma carência de dados em relação às respostas cardiovasculares agudas do ER, realizado com alta velocidade de contração muscular, na população idosa. Além disso, os efeitos da utilização de diferentes estratégias como a de protocolos contínuos e descontínuos no treinamento de potência, na população idosa, precisam ser investigados. Dessa forma, o presente estudo teve por objetivo comparar as respostas cardiovasculares agudas da FC, PA e DP entre três diferentes protocolos de treinamento resistido (contínuo vs descontínuos), realizado com alta velocidade de contração muscular, em mulheres idosas destreinadas.

\section{PROCEDIMENTOS METODOLÓGICOS}

\section{Sujeitos}

A amostra do presente estudo foi composta por 12 mulheres idosas voluntárias, com idade entre 60 a 70 anos (62,6 $\pm 2,9$ anos de idade; massa corporal $57,3 \pm 7,9 \mathrm{~kg}$; estatura $1,53 \pm 0,07 \mathrm{~m}$; gordura corporal $27,8 \pm 2,6 \%$ ). As voluntárias estavam sem praticar ER há pelo menos um ano. As idosas não faziam uso de qualquer medicação que pudesse alterar as respostas cardiovasculares agudas durante o exercício. Antes de iniciar o estudo, solicitou-se de cada participante a assinatura de um termo de consentimento livre e esclarecido, conforme a orientação do comitê e da resolução no 196/96 do Conselho Nacional de Saúde. O projeto foi aprovado pelo Comitê de Ética em Pesquisa da Universidade Católica de Brasília/ DF (CEP/UCB n ${ }^{\circ}$ 025/2004).

Após a apresentação da liberação médica para realização de exercícios, da assinatura do termo de consentimento e dos esclarecimentos dos objetivos da pesquisa, foi realizado um estudo piloto com quatro voluntárias.

\section{Teste de 10 repetições máximas (10RM)}

A carga para o exercício supino horizontal e leg press $45^{\circ}$ foi estipulada durante o teste de 10-RM. A determinação da carga para 10-RM, nos dois exercícios, foi feita no mesmo dia, com um intervalo mínimo de 30 minutos entre os testes. A ordem de execução dos exercícios para determinação da carga de 10-RM foi aleatória. Para determinação da confiabilidade do teste de 10-RM, nos dois exercícios, foi realizado o re-teste com, no mínimo, cinco e no máximo, 10 dias após o teste (tabela 1).

$\mathrm{O}$ teste de 10RM foi realizado seguindo as seguintes recomendações adaptadas de Kraemer e Fry ${ }^{14}$ : 
1) aquecimento de 5 a 10 repetições com cargas de 40 a 60\% de 1RM estimada; 2) descanso de um minuto, seguidos de três a cinco repetições com $60 \%$ de 1 RM estimada e um descanso de três minutos; 3) incremento do peso $(\sim 5 \%)$ tentando alcançar as 10RM em três a cinco tentativas, usando cinco minutos de intervalo entre uma tentativa e outra; 4) o valor registrado foi o de 10 repetições, com o peso máximo levantado na última tentativa bem sucedida.

Para uma maior segurança das voluntárias, durante a execução do teste de 10-RM, além do intuito de se obterem valores mais representativos das respostas agudas ao ER, as voluntárias realizaram um treinamento, durante três semanas (fase de adaptação), nos exercício supino horizontal e leg pres $45^{\circ}$ antes da aplicação dos testes de 10RM. A fase de adaptação consistiu em realizar os exercícios nos protocolos utilizados no estudo, nos mesmos equipamentos. $\mathrm{Na}$ fase de adaptação foram realizadas três séries de 10 repetições, três vezes por semana em dias não consecutivos.

\section{Protocolo experimental}

Durante a realização dos dois exercícios, na coleta das variáveis, as voluntárias foram orientadas quanto à realização correta da técnica do movimento, sendo sempre monitoradas por um avaliador. Além disso, todas as voluntárias eram desencorajadas a realizarem a manobra de valsalva, sendo orientadas a expirar durante a fase concêntrica do movimento. Esse procedimento foi treinado durante a fase de adaptação.

As variáveis $\mathrm{FC}, \mathrm{PA}$ e DP foram coletadas no PC, PD5 e PD15. O PC foi caracterizado pela realização do ER com 10 repetições contínuas, o PD5 por uma pausa entre a quinta e sexta repetições de cinco segundos e o PD15 por uma pausa de 15 segundos entre a quinta e sexta repetições. Todos os protocolos foram realizados com a carga encontrada no teste de 10-RM.

Para uma maior confiabilidade e evitar maiores comprometimentos de validade interna do estudo, foi realizada a reprodutibilidade da medida da pressão arterial sistólica - PAS (tabela1) durante a fase de adaptação. Além disso, a FC e a PA foram coletadas por um avaliador experiente e treinado.

Os três protocolos foram realizados com alta velocidade de contração muscular. Para tal, as voluntárias foram instruídas a realizarem a fase concêntrica do movimento com a máxima velocidade possível e 2-3 segundos para a fase excêntrica.

A realização do leg press $45^{\circ}$ e do supino horizontal para a coleta das variáveis no PC, PD5 e PD15 foi feita em três dias, com um intervalo mínimo de sete e no máximo de 10 dias entre os testes. A escolha dos protocolos (PC, PD5 ou PD15), em cada dia, para coleta das variáveis, nos dois exercícios, foi contrabalanceada. A ordem de execução dos exercícios, para a coleta das variáveis, foi contrabalanceada. Após a realização do primeiro exercício, a voluntária descansava, no mínimo, 10 minutos para realizar o segundo exercício. Antes da realização do segundo exercício, aferia-se novamente a PAS. A PAS não poderia estar acima ou abaixo de um desvio padrão do valor médio do repouso para a realização do segundo exercício ${ }^{15}$. O valor médio de repouso da PAS foi calculado pela média aritmética das pressões tomadas durante a fase de adaptação.

Antes de iniciar o experimento, a voluntária permanecia sentada por 10 minutos para aferição da FC e da PAS de repouso. Caso a PAS estivesse maior que um desvio padrão do valor médio determinado durante a fase de adaptação, os dados não eram coletados. Em seguida, as voluntárias fizeram 10 minutos de aquecimento orgânico em cicloergômetro, mantendo entre 50 a 60 rpm, com uma carga de 25 watts. Após o aquecimento no clicloergômetro, fez-se uma série de aquecimento específico de 10 repetições a 50\% da carga de 10RM, no aparelho no qual seria realizado o exercício (leg press $45^{\circ}$ e supino horizontal). Três minutos após o aquecimento, foram realizadas três séries de 10-RM, com um intervalo de dois minutos entre as séries para a coleta das variáveis.

Anotou-se o maior valor de FC, no final de cada série, nos dois exercícios, ou imediatamente após. A FC foi aferida pelo cardiofrequecímetro modelo S810 $\left(\right.$ Polar $\left.^{\circledR}\right)$. A PA foi aferida imediatamente ao final de cada série no exercício supino horizontal. Para tal, o esfigmomanômetro começava a ser insuflado na nona repetição $0^{16}$. Dessa forma, ao final da última repetição o esfigmomanômetro estava totalmente insulflado o que permitia e leitura da PA imediatamente após o término de cada série. No exercício leg press $45^{\circ}$, a PA foi aferida durante a execução do exercício, nas três séries, entre a antepenúltima e última repetição. Considerou-se como valor sistólico, a primeira fase do som de Korotkoff ${ }^{16}$. A PA foi aferida pelo método auscultatório, com aparelho de pressão coluna de mercúrio (Glicomed $^{\circledR}$ ) e estetoscópio (Rappaport $\left.{ }^{\circledR}\right)$. O duplo produto (DP) foi medido pelo produto da FC com a pressão arterial sistólica (PAS).

\section{Tratamento estatístico}

Para verificação da normalidade dos dados, foi feito o teste Shapiro Wilk. Em seguida, utilizou-se 
o cálculo da média e desvio padrão para apresentação das variáveis estudadas. Para determinação da possível diferença entre as medidas do teste e re-teste de 10-RM e da PAS, foi utilizado o teste $t$ de student para amostras dependentes. A confiabilidade das medidas de teste e re-teteste do teste de 10-RM e da PAS foi realizada pelo Coeficiente de Concordância de Kendall (W). Na comparação dos valores médios de FC, PAS e DP entre os PC, PD5 e PD15, utilizou-se a análise de variância fatorial 3 $\mathrm{x} 4$ [protocolos (PC, PD5, PD15) x tempo (repouso, $1^{\mathrm{a}}, 2^{\mathrm{a}}, 3^{\mathrm{a}}$ séries)] de medidas repetidas (within $\mathrm{x}$ within). Os dados foram analisados por meio do programa estatístico (Statistical Package for the Social Sciences - SPSS) 13.00 para Windows. Para determinar as possíveis diferenças estatisticamente significativas da ANOVA, utilizou-se o teste posthoc Least Significant Difference (LSD).

\section{RESULTADOS}

A tabela 1 apresenta o resultado da reprodutibilidade teste e re-teste de 10RM e da PAS. Não foram encontradas diferenças significativas $(p<0,01)$ no teste $t$ de student, para amostras dependentes, entre o teste e re-teste de 10RM e na medida de PAS.

As tabelas 2 e 3 apresentam os resultados da FC, PAS e DP nos PC, PD5 e PD15 no exercício supino horizontal e leg press $45^{\circ}$, respectivamente.

A tabela 2 demonstra que no exercício supino horizontal a FC, a PAS e o DP, no repouso, foram significativamente menores que a $1^{a}, 2^{a}$ e $3^{a}$ séries, respectivamente. $\mathrm{A} 1^{\mathrm{a}}$ série foi significativamente menor que a $2^{a}$ e $3^{a}$ séries na PAS e no DP, nos três protocolos e na FC, apenas no PC. Na comparação entre os três protocolos a FC foi significativamente menor no PD5 em comparação ao PC, nas três séries e o PD15 foi menor em relação ao $\mathrm{PC}$ na $2^{\mathrm{a}}$ e $3^{\mathrm{a}}$ séries. A PAS não apresentou diferença significativa entre os três protocolos. O DP foi significativamente menor no PD5 e PD15 em comparação ao PC na $2^{\mathrm{a}}$ e $3^{\mathrm{a}}$ séries.

A tabela 3 demonstra que no exercício leg press $45^{\circ}$ a FC, a PAS e o DP no repouso foram significativamente menores que a $1^{\mathrm{a}}, 2^{\mathrm{a}}$ e $3^{\mathrm{a}}$ séries, respectivamente. $\mathrm{A} 1^{\mathrm{a}}$ série foi significativamente menor que a $2^{a}$ e $3^{a}$ séries na PAS nos três protocolos e no DP no PD5 e PD15. Já no PC o DP na $1^{a}$ série foi significativamente menor apenas em relação à $3^{a}$ série. $\mathrm{Na} \mathrm{FC}$, na $1^{\mathrm{a}}$ série, apenas o PD5 foi significativamente menor em relação à $3^{\mathrm{a}}$ série. Na comparação entre os três protocolos, apenas o PD5 foi significativamente menor em relação ao PC na $3^{a}$ série na FC, na PAS e no DP.

\section{DISCUSSÃO}

No presente estudo, a FC, PAS e DP foram significativamente maiores nas três séries realizadas, em relação ao repouso, no exercício leg press $45^{\circ}$ e no

Tabela 1. Resultados da reprodutibilidade do teste e re-teste de 10-RM e da pressão arterial sistólica $(\mathrm{n}=12)$

\begin{tabular}{lcccc}
\hline Exercícios & Teste $(\mathrm{Kg})$ & Re-teste $(\mathrm{Kg})$ & $\mathrm{t}$ & $\mathrm{W}$ \\
\hline Leg Pres 45 & $65,08 \pm 10,61$ & $66,00 \pm 10,85$ & $-1,081$ & $0,97^{*}$ \\
Supino Horizontal & $19,16 \pm 3,06$ & $19,66 \pm 3,28$ & $-1,483$ & $0,97^{*}$ \\
Pressão Arterial Sistólica & $122,08 \pm 3,96$ & $122,50 \pm 4,52$ & $-1,587$ & $0,96^{*}$ \\
\hline
\end{tabular}

$\mathrm{RM}=$ repetição máxima; $\mathrm{W}=$ Coeficiente de Concordância de Kendall; ${ }^{*} \mathrm{p}<0,05$ teste versus re-teste

Tabela 2. Resultados da FC, PAS e DP nos PC, PD5 e PD15 no exercício supino horizontal.

\begin{tabular}{|c|c|c|c|c|c|}
\hline & & Repouso & $1^{a}$ série & $2^{\mathrm{a}}$ série & $3^{\mathrm{a}}$ série \\
\hline \multirow[t]{3}{*}{$\mathrm{FC}$} & PC & $79,1 \pm 10,8^{*}$ & $98,0 \pm 13,8^{\ddagger}$ & $100,2 \pm 12,9$ & $100,6 \pm 14,1$ \\
\hline & PD5 & $79,8 \pm 9,0^{*}$ & $93,9 \pm 12,9^{\dagger}$ & $94,7 \pm 13,3^{\dagger}$ & $95,3 \pm 13,2^{\dagger}$ \\
\hline & PD15 & $79,0 \pm 8,4^{*}$ & $94,7 \pm 7,8$ & $94,9 \pm 9,0^{\dagger}$ & $95,2 \pm 9,6^{\dagger}$ \\
\hline \multirow[t]{3}{*}{ PAS } & PC & $117,5 \pm 6,5^{*}$ & $132,5 \pm 11,3^{\ddagger}$ & $148,3 \pm 14,0$ & $147,5 \pm 14,8$ \\
\hline & PD5 & $120,4 \pm 2,5^{*}$ & $134,5 \pm 8,3^{\ddagger}$ & $143,3 \pm 6,1$ & $145,0 \pm 9,0$ \\
\hline & PD15 & $116,6 \pm 4,9^{*}$ & $133,7 \pm 9,3^{\ddagger}$ & $141,6 \pm 8,3$ & $144,5 \pm 13,3$ \\
\hline \multirow[t]{3}{*}{ DP } & PC & $9285,8 \pm 1241,9^{*}$ & $13055,8 \pm 2593,7^{\ddagger}$ & $14916,6 \pm 2683,1$ & $14919,1 \pm 2997,6$ \\
\hline & PD5 & $9617,0 \pm 1152,7^{*}$ & $12643,7 \pm 1891,1^{\ddagger}$ & $13604,5 \pm 2169,3^{\dagger}$ & $13843,3 \pm 2189,3^{\dagger}$ \\
\hline & PD15 & $9225,0 \pm 1135,0^{*}$ & $12665,0 \pm 1240,3^{\ddagger}$ & $13441,6 \pm 1404,9^{\dagger}$ & $13740,8 \pm 1584,5^{\dagger}$ \\
\hline
\end{tabular}

$\mathrm{p}<0,05 v s 1^{1 \mathrm{a}}, 2^{\mathrm{a}}$, e $3^{\mathrm{a}}$ séries; ${ }^{\ddagger} \mathrm{p}<0,05$ vs $2^{\mathrm{a}}$ e $3^{\mathrm{a}}$ séries; $^{\dagger} \mathrm{p}<0,05$ vs PC.

$\mathrm{FC}=$ frequência cardíaca; $\mathrm{PAS}=$ pressão arterial sistólica; $\mathrm{DP}=$ duplo produto; $\mathrm{PC}=$ protocolo contínuo; $\mathrm{PD} 5=$ protocolo descontínuo 5s; PD15 = protocolo descontínuo 15s. 
Tabela 3. Resultados da FC, PAS e DP nos PC, PD5 e PD15 no exercício leg press 45o.

\begin{tabular}{|c|c|c|c|c|c|}
\hline & & Repouso & $1^{a}$ série & $2^{a}$ série & $3^{a}$ série \\
\hline \multirow[t]{2}{*}{$\mathrm{FC}$} & PC & $79,0 \pm 9,4^{*}$ & $106,9 \pm 12,2$ & $108,5 \pm 11,3$ & $111,2 \pm 13,2$ \\
\hline & PD5 & $74,5 \pm 10,9^{*}$ & $103,0 \pm 11,0^{\S}$ & $104,1 \pm 11,3$ & $105,7 \pm 11,9^{\dagger}$ \\
\hline \multirow[t]{2}{*}{ PAS } & PC & $120.0 \pm 5.6^{*}$ & $154.1 \pm 22.3^{\ddagger}$ & $163.3 \pm 13.7$ & $174.1 \pm 15.0$ \\
\hline & PD5 & $115.8 \pm 5.1^{*}$ & $149.1 \pm 14.4^{\ddagger}$ & $155.4 \pm 11.5$ & $161.2 \pm 11.3^{\dagger}$ \\
\hline \multirow[t]{3}{*}{ DP } & $\mathrm{PC}$ & $9459,0 \pm 1233,5^{*}$ & $16792,5 \pm 3227,3^{\S}$ & $17758,3 \pm 2577,5$ & $19391,6 \pm 2815,5$ \\
\hline & PD5 & $8632,5 \pm 1332,9^{*}$ & $15423,3 \pm 2751,0^{\ddagger}$ & $16720,8 \pm 3077,6$ & $16884,1 \pm 2931,9^{\dagger}$ \\
\hline & PD15 & $9082,0 \pm 1249,7^{*}$ & $16148,3 \pm 2729,3^{\ddagger}$ & $17564,1 \pm 3107,6$ & $18901,6 \pm 4039,7$ \\
\hline
\end{tabular}

$\mathrm{p}<0,05$ vs $1^{\mathrm{a}}, 2^{\mathrm{a}}$, e $3^{\mathrm{a}}$ séries; ${ }^{\ddagger} \mathrm{p}<0,05$ vs e $2^{\mathrm{a}}$ e $3^{\mathrm{a}}$ séries; ${ }^{\S} \mathrm{p}<0,05$ vs $3^{\mathrm{a}}$ série; ${ }^{+} \mathrm{p}<0,05$ vs $\mathrm{PC}$.

$\mathrm{FC}=$ frequência cardíaca; PAS = pressão arterial sistólica; DP = duplo produto; PC = protocolo contínuo; PD5 = protocolo descontínuo 5s; PD15 = protocolo descontínuo 15s.

supino horizontal. Vários outros estudos também apontaram para o mesmo comportamento da FC, PAS e DP no ER ${ }^{13,15,17,18}$. As alterações cardiovasculares durante o ER podem ser explicadas pelos receptores musculares que são sensíveis às alterações metabólicas e mecânicas impostas pelo exercício $^{19,20}$. Quando o exercício é iniciado, esses receptores, através de impulsos aferentes, enviam sinais à medula espinhal que o conduzem até as áreas cardiovasculares do sistema nervoso central (SNC). Como resultado, a atividade parassimpática para o coração é diminuída e a atividade simpática para o coração é aumentada ${ }^{21}$. Associado aos receptores musculares, sinais neurais originados no cérebro, através do reflexo barorreceptor nas artérias aórtica e carótida, também elevam a atividade excitatória do sistema nervoso simpático ${ }^{22}$. Além disso, durante uma sessão de exercício há um aumento da concentração plasmática de angiotensina 11 , a qual contribui para o efeito pressor do exercício através de uma facilitação da liberação de norepinefrina pelos neurônios pós-ganglionares simpáticos ${ }^{23}$. A combinação desses fatores leva ao aumento da FC, PAS e DP no ER.

Outro mecanismo que explicaria maiores valores de FC, PAS e DP, no ER, está associado à liberação de metabólitos pelo músculo ativo como, por exemplo, o lactato. Esses metabólitos ativariam os quimiorreceptores que mantêm comunicação direta com o centro de controle cardiovascular que, por sua vez, também aumentaria a $\mathrm{PA}^{21,24}$. Ainda, a elevação do hidrogênio e da pressão do gás carbônico induzidas pelo exercício provoca ativação do centro bulbar, exacerbando as respostas cardiovasculares agudas ao exercício ${ }^{25}$.

Com relação à comparação entre os três protocolos estudados (PC, PD5 e PD15) no exercício supino horizontal, a FC foi significativamente me- nor no PD5 em relação ao $\mathrm{PC}$ na $1^{\mathrm{a}}, 2^{\mathrm{a}}$ e $3^{\mathrm{a}}$ séries e o PD15 foi significativamente menor em relação ao PC na $2^{\mathrm{a}}$ e $3^{\mathrm{a}}$ séries. A PAS foi semelhante entre os três protocolos nas três séries. O DP foi significativamente menor em relação ao $\mathrm{PC}$ na $2^{\mathrm{a}} \mathrm{e} 3^{\mathrm{a}}$ séries. No exercício leg press $45^{\circ}$, a FC, a PAS e o DP foram significativamente menores no PD5 em relação ao PC apenas na $3^{\mathrm{a}}$ série dos três protocolos.

O comportamento de menor estresse cardiovascular observado com maior frequência no exercício supino horizontal que no exercício leg press $45^{\circ}$ nos PD5 e PD15 pode ser devido a um período de menor oclusão arterial imposto por estes protocolos, já que os dois protocolos foram realizados com uma pausa de cinco e 15 segundos, respectivamente, entre a quinta e sexta repetições, ainda que os três protocolos estudados tivessem sido realizados com o mesmo volume de trabalho ${ }^{13,17}$.

Outros estudos confirmam essa hipótese ao demonstrarem uma forte relação entre a duração do período de tensão muscular no ER e uma maior sobrecarga cardiovascular ${ }^{26}$. Outro fato é que os intervalos de cinco (PD5) e 15 segundos (PD15), nas três séries realizadas, permitiria uma melhor remoção dos metabólicos (lactato) produzidos durante as cinco primeiras repetições. Com isso, nas cinco últimas repetições os quimiorreceptores, que são ativados pelo lactato, levariam a uma menor atividade do centro de controle cardiovascular, desencadeando menores valores de FC, PAS e DP24. Além disso, os protocolos descontínuos parecem promover uma melhor recuperação da fosfocreatina, levando a uma menor glicólise anaeróbia no músculo ativo, o que desencadearia menores valores de lactato sanguíneo ${ }^{10}$.

Apesar dos menores valores da FC, PAS e DP nos protocolos descontínuos, esse comportamento não foi consistente nas três séries realizadas nos dois 
exercícios. No supino horizontal, a FC na $1^{\text {a }}$ série foi menor no PD5 em relação ao PC e o mesmo não foi observado no PD15. No leg press $45^{\circ}$, na $3^{\text {a }}$ série a FC, a PAS e o DP no PD5 foram menores em relação ao $\mathrm{PC}$ e o mesmo não foi observado no PD15. Nossa hipótese era que o protocolo descontínuo de maior pausa (PD15) desencadeasse menores respostas cardiovasculares agudas no ER, contudo, o PD5 apresentou menores valores, como descrito anteriormente. A resposta para esse comportamento pode ser explicada pela posição corporal assumida pelas voluntárias durante as pausas de cinco e quinze segundos, na execução das três séries. As voluntárias mantinham os braços (supino horizontal) e as pernas (leg press $45^{\circ}$ ) em contração isométrica para manter a mesma posição de execução do movimento após as pausas, sem sustentar a carga. O maior período de contração isométrica do PD15 pode ter exacerbado as respostas cardiovasculares agudas em relação ao PD5, isto em virtude do maior período de contração isométrica impor maiores respostas cardiovasculares agudas ao $\mathrm{ER}^{27}$.

A FC está inversamente relacionada ao tamanho do grupamento muscular envolvido no ER, ou seja, grupos musculares de menor tamanho impõem uma maior resistência vascular periférica (RVP) ao fluxo sanguíneo. Como consequência, a FC é aumentada na tentativa de compensar a maior $\mathrm{RVP}^{28}$. Esse mecanismo é visto no presente estudo, mesmo sem ter sido feito um teste estatístico para comparar os valores da FC entre os exercícios supino horizontal e leg press $45^{\circ}$, observa-se no exercício de menor tamanho muscular (supino horizontal) valores mais elevados de FC. Além disso, as pausas de cinco (PD5) e quinze (PD15) segundos seriam suficientes para reduzir a RVP ao fluxo sanguíneo, diminuindo a elevação compensatória da FC.

Os PD5 e PD15 foram significativamente menores que o $\mathrm{PC}$ nas últimas séries ( $2^{\mathrm{a}}$ ou $3^{\mathrm{a}}$ séries) dos dois exercícios. Tal fato pode ser explicado pela maior demanda metabólica imposta ao término do exercício. $\mathrm{O}$ estresse metabólico do acúmulo das séries executadas provoca um maior recrutamento de unidades motoras, levando a maiores respostas cardiovasculares agudas ao ER ${ }^{29}$. Nesse sentido, as pausas de cinco e 15 segundos contribuiriam para atenuar, pelo menos em parte, o maior estresse cardiovascular decorrente do recrutamento de novas unidades motoras.

Corroborando o presente estudo, Veloso et al..$^{13}$ relataram valores significativamente menores do DP no protocolo fracionado (quatro séries de seis repetições), em relação a protocolo contínuo (duas séries de 12 repetições). Contudo os mesmo autores apontaram semelhanças nos valores da FC entre o protocolo fracionado e contínuo. Uma possibilidade da divergência entre os dois estudos na FC é a diferença no protocolo utilizado. Outra possível explicação pode ser atribuída ao fato do estudo de Veloso et al. ${ }^{13}$ não ter controlado a velocidade de contração muscular.

Coelho et al. ${ }^{10}$ também compararam a resposta da FC em um protocolo contínuo (12RM ininterruptamente) com o protocolo descontínuo (12RM com uma pausa de 15 segundos entre a sexta e sétima repetições) realizados com alta velocidade de contração muscular em jovens. $\mathrm{O}$ protocolo descontínuo apresentou valor médio de FC significativamente menor em relação ao protocolo contínuo. Esse comportamento também parece existir na população idosa, o que pode ser observado nos resultados encontrados no presente estudo.

Contrariando os resultados do presente estudo, Polito et al. ${ }^{5}$ observaram valores médios maiores de PA e DP na extensão de joelho unilateral fracionada comparada à execução de forma contínua. A controvérsia entre os dois estudos pode ser atribuída ao delineamento metodológico. No estudo de Polito et al. ${ }^{5}$ foram apenas dois segundos de pausa entre a quarta e quinta repetições e maior intensidade de treinamento (8-RM).

No presente estudo, a PA foi aferida pelo método auscultatório, o que pode ter subestimado os valores encontrados ${ }^{30}$. O método padrão-ouro considerado pela literatura é a medida intra-arterial. No entanto, por ser um método invasivo, pode acarretar riscos elevados para os indivíduos como, por exemplo, dor, espasmo arterial, trombose, estenose, síncope vaso-vagal, hemorragia, etc. Além disso, a medida intra-arterial é muito mais sensível aos pulsos de pressões durante o exercício e menos à detecção do fluxo, o que também pode levar a uma quantificação errada da $\mathrm{PA}^{30}$.

Os valores absolutos da PA, subestimados pelo método auscultatório, em exercícios mais intensos tendem a manter relação entre medidas realizadas em diferentes momentos ${ }^{30}$. Partindo do princípio de que o erro na aferição da PA pelo método auscultatório acontece de forma sistemática, isso parece não comprometer os resultados desse estudo, visto que o principal objetivo é comparar os valores da PA em diferentes situações do ER.

Conclui-se que a utilização dos protocolos com as pausas de cinco (PD5) e 15 segundos (PD15) proporcionaram uma menor sobrecarga cardiovascular (FC, PAS e DP) em relação ao PC no ER realizado 
com alta velocidade de contração muscular em idosas adaptadas ao ER. Pode-se inferir, também, que, em alguns momentos, principalmente, no exercício de membro inferior, o PD5 parece apresentar um menor estresse cardiovascular (FC, PAS e DP) quando comparado com outros protocolos. Dessa forma, a adoção de protocolos descontínuos, com pausas de cinco segundos, parece ser uma estratégia interessante para uma maior segurança na prescrição do ER da população idosa.

\section{REFERÊNCIAS BIBLIOGRÁFICAS}

1. Baumgartner RN, Koehler KM, Gallagher D, Romero L, Heymsfield SB, Ross RR, et al. Epidemiology of sarcopenia among the elderly in New Mexico. Am J Epidemiol 1998;147(8):755-763.

2. Hakkinen K, Kraemer WJ, Pakarinen A, TriplettMcBride T, McBride JM, Hakkinen A, et al. Effects of heavy resistance/power training on maximal strength, muscle morphology, and hormonal response patterns in 60-75-year-old men and women. Can J Appl Physiol 2002;27(3):213-231.

3. Izquierdo M, Aguado X, Gonzalez R, Lopez JL, Hakkinen K. Maximal and explosive force production capacity and balance performance in men of different ages. Eur J Appl Physiol Occup Physiol 1999;79(3):260-267.

4. Bottaro M, Machado S, Nogueira W, Scales R, Veloso J. Effect of High versus Low-Velocity Resistance Training on Muscular Fitness and Functional Performance in Older Men. Eur J Appl Physiol 2007;99(3):257-264.

5. Polito MD, Simão R, Lira VA, Nóbrega ACL, Farinatti PTV. Série fracionada da extensão do joelho porporciona maiores respostas cardiovasculares que séries contínuas. Arq Bras Cardiol 2008;90(6):382-387.

6. Henwood TR, Taaffe DR. Improved physical performance in older adults undertaking a short-term program of high-velocity resistance training. Gerontology 2005;51(2):108-115.

7. Hudra KV, Hicks AL, Mccartney N. Training for muscle power in older adults: effects on functional abilities. Can J Appl Physiol 2003;28(2):178-189.

8. McCartney N. Acute responses to resistance training and safety. Med Sci Sports Exerc 1999;31(1):31-37.

9. Karlsdottir AE, Foster C, Porcari JP, Palmer-Mclean K, White-Kube R, Backes RC. Hemodynamic response during aerobic and resistance exercise. J Cardiopulm Rehabil 2002;22(3):170-177.

10. Coelho CW, Hamar D, Araujo CGS. Physiological responses using 2 high-speed resistance training protocols. J Strength Cond Res 2003;17(2):334-337.

11. Keeler LK, Finkelstein LH, Miller W, Fernhall B. Early - Phase adaptations of traditional - speed vs. superslow resistance training on strength and aerobic capacity in sedentary individuals. J Strength Cond Res 2001;15(3):309-314.
12. Bermon S, Rama D, Dolisi C. Cardiovascular tolerance of healthy elderly subjects to weight-lifting exercises. Med Sci Sports Exerc 2000;32(11):1845-1848.

13. Veloso U, Monteiro W, Farinatti P. Exercícios contínuos e fracionados provocam respostas cardiovasculares similares em idosas praticantes de ginástica? Rev Bras Med Esporte 2003;9(2):78-84.

14. Kraemer WJ, Fry AC. Strength Testing: Development and Evaluation of Methodology In Physiological assessment of human fitness. Champaign: Human Kinetics; 1995.

15. Macdonald JR, Macougall JD, Hogben C. The effects of exercise intensity on post exercise hypotension. J Hum Hypertens 1999;13(8):527-531.

16. Polito MD, Farinatti PTV. Considerações sobre a medida da pressão arterial em exercícios contra-resistência. Rev Bras Med Esporte 2003;9(1):25-33.

17. Polito MD, Rosa CC, Schardong P. Respostas cardiovasculares agudas na extensão do joelho realizada em diferentes formas de execução. Rev Bras Med Esporte 2004;10(3):173-176.

18. Carvalho J, Mota J, Soares JMC. Exercício de força versus exercícios aeróbios: tolerância cardiovascular em idosos. Rev Port Cardiol 2003;22(11):1315-1330.

19. Gallagher KM, Fadel PJ, Smith SA, Stromstad M, Ide $\mathrm{K}$, Secher NH, et al. The interaction of central command and the exercise pressor reflex in mediating baroreflex resetting during exercise in humans. Exp Physiol 2006;91(1):79-87.

20. Joyner MJ. Barorecptor function during exercise: resetting the record. Exp Physiol 2006;91(1):27-36.

21. Mitchell JM. Cardiovascular control during exercise: Central and reflex neural mechanisms. Am J Cardiol 1985;55(10):34D-41D.

22. Guyenet PG. The sympathetic control of blood pressure. Nat Rev Neurosci 2006;7(5):335-336.

23. Warren JH, Lewis W, Wraa CE, Stebbins CL. Central and peripheral effects of angiotensina II on the cardiovascular response to exercise. J Cardiovasc Pharmacol 2001;38(5):693-705.

24. Mitchell JH, Schibye B, Payne FC, Saltin B. Response of arterial blood pressure to static exercise in relation to muscle mass, force development, and electromyography activity. Circ Res 1981(6);48:170-175.

25. Porto M, Orsatti FL, Santos MDB, Burini RC. Impacto do exercício muscular exaustivo sobre indicadores sanguíneos em praticantes de musculação. Rev Bras Cineantropom Desempenho Hum 2008;10(3):230-236.

26. Rozenek R, Rosenau L, Rosenau P, Stone MH. The effect of intensity on heart rate and blood lactate response to resistance exercise. J Strength Cond Res 1993;7(1):51-54.

27. Smolander J, Aminoff T, Korhonen I, Tervo M, Shen $\mathrm{N}$, Korhonen $\mathrm{O}$, et al. Heart rate and blood pressure responses to isometric exercise in young and older men. Eur J Appl Physiol 1998,77(5):439-444. 
28. Sahlin K, Freyschuss U, Hultman E, Sjoholm H. Effect os short-term $\mathrm{CO} 2$ breathing on the $\mathrm{CO} 2$ content and intracellular $\mathrm{pH}$ in skeletal muscle of man. Clin Physiol 1981,1(5):495-502.

29. Macdougall JD, Tuxen D, Sale DG, Moroz JR, Sutton JR. Arterial blood pressure response to heavy resistance exercise. J. Appl Physiol 1985,58(3):785-790.

30. Leite T, Farinnati PTV. Estudo da frequencia cardísca, pressão arterial e duplo produto em exercícios resistidos diversos para grupamentos musculares semelhantes. Rev Bras Fisiol Exerc 2003,2(1):68-88.

\section{Endereço para correspondência}

Rodrigo Pereira da Silva

Rua Professor Paulo de Magalhães Gomes, n³99,

Bairro Bauxita

CEP: 35400-000. Ouro Preto. MG. Brasil.

E-mail: rodrigosilva@cedufop.ufop.br 